\title{
Outbreak of plasmid-mediated NDM-1-producing Klebsiella pneumoniae ST105 among neonatal patients in Yunnan, China
}

\author{
Rui Zheng ${ }^{1,2,3 \dagger}$, Qian Zhang ${ }^{4 \dagger}$, Yidan Guo ${ }^{5}$, Yue Feng ${ }^{1,2}$, Li Liu ${ }^{1,2}$, Amei Zhang ${ }^{1,2}$, Yue Zhao ${ }^{1,2}$, Xiaoyu Yang ${ }^{1,2}$ \\ and Xueshan $\mathrm{Xia}^{1,2^{*}}$
}

\begin{abstract}
Background: In the past decade, the carbapenemase-producing Enterobacteriaceae (CPE) have been reported worldwide. Emergence of carbapenemase-producing strains among Enterobacteriaceae has been a challenge for treatment of clinical infection. The present study was undertaken to investigate the characteristics of carbapenemresistant Klebsiella pneumoniae recovered from an outbreak that affected 17 neonatal patients in neonatal intensive care unit (NICU) of Kunming City Maternal and Child health Hospital, which is located in the Kunming city in far southwest of China.

Methods: Minimum inhibitory concentrations (MICs) for antimicrobial agents were determined according to the guidelines of the Clinical and Laboratory Standards Institute (CLSI); Modified Hodge test and Carba-NP test were preformed to identified the phenotypes of carbapenemases producing; To determine whether carbapenem resistance was transferable, a conjugation experiment was carried out in mixed broth cultures; Resistant genes were detected by using PCR and sequencing; Plasmids were typed by PCR-based replicon typing method; Clone relationships were analyzed by using multilocus-sequence typing (MLST) and pulsed field gel electrophoresis (PFGE).
\end{abstract}

Results: Eighteen highly carbapenem-resistant Klebsiella pneumoniae were isolated from patients in NICU and one carbapenem-resistant $K$. pneumoniae isolate was detected in incubator water. All these isolates harbored bla $a_{\mathrm{NDM}-1}$. Moreover, other resistance genes, viz., bla $a_{\mathrm{IMP}-4}, b a_{\mathrm{SHV}-1}, b / a_{\mathrm{TEM}-1}, b / a_{\mathrm{CTX}-\mathrm{M}-15}$, anrS1, anrB4, and aacA4 were detected. The bla $a_{\mathrm{NDM}-1}$ gene was located on a ca. $50 \mathrm{~kb}$ IncFI type plasmid. PFGE analysis showed that NDM-1-producing K. pneumoniae were clonally related and MLST assigned them to sequence type 105.

Conclusions: NDM-1 producing strains present in the hospital environment pose a potential risk and the incubator water may act as a diffusion reservoir of NDM-1- producing bacteria. Nosocomial surveillance system should play a more important role in the infection control to limit the spread of these pathogens.

Keywords: CPE, NDM-1, ST105, China

\section{Background}

Gram-negative bacilli are the most important cause of healthcare associated infections [1]. Among these, Enterobacteriaceae continue to be an important cause

\footnotetext{
*Correspondence: oliverxia2000@aliyun.com

${ }^{\dagger}$ Rui Zheng and Qian Zhang contributed equally to this paper

${ }^{2}$ Faculty of Life Science and Technology, Kunming University of Science and Technology, No. 727 Jing Ming Road, Chenggong District, Kunming, Yunnan, China

Full list of author information is available at the end of the article
}

of such infections [2], particularly the carbapenemaseproducing Enterobacteriaceae (CPE) in developing countries, such as China [3]. In the past decade, the CPEs have been reported worldwide, including KPC-, GES-, VIM-, IMP-, GIM-, NDM- and OXA- types [4]. Among them, the NDM-type carbapenemase is a novel metalo-betalactamase that was identified for the first time in 2008 [5]. NDM-1 carbapenemase belongs to class B of Ambler $\beta$-lactamases, and efficiently hydrolyses a broad range of $\beta$-lactams, including penicillins, cephalosporins, and 
carbapenems, except for aztreonam. Up to this day, the emergence of carbapenemase-producing strains among Enterobacteriaceae has been a challenge for treatment of clinical infection [6].

Plasmid-mediated drug resistance is one of the most serious problems in the treatment of infectious diseases due to the horizontal transfer of plasmids account for the dissemination of resistance genes and the emergence of drug resistant strains [7, 8]. Carbapenemase-producing strains are most often associated with many non- $\beta$ lactam-resistance genes, because of their locations on plasmids [9], which made therapeutic options for infections were very limited.

Klebsiella pneumoniae was a leading cause of nosocomial infections and spread rapidly in health care settings due to efficiency of colonization and rapid development of resistance to a wide range of antimicrobials [10]. Recently, K. pneumoniae harboring bla $_{\mathrm{NDM}-1}$ were emergencing in China, which should pay great attention $[11,12]$. Therefore, investigation of the molecular characteristics of NDM-1-producing K. pneumoniae is critical. Here, we identified 19 K. pneumoniae harboring $b l a_{\mathrm{NDM}-1}$, the transmission of these NDM-1-producing $K$. pneumoniae among neonatal patients at Kunming City Maternal and Child health Hospital was delineated in this study.

\section{Methods}

\section{Bacterial isolates}

Kunming City Maternal and Child health Hospital was a 200-bed tertiary care community health facility in the provincial capital, Kunming City. Carbapenem-resistant Enterobacteriaceae (CRE) isolates were rare in this facility prior to this outbreak. On January 22, 2014, one K. pneumoniae strain (M1) was isolated from a sputum specimen, obtained from a neonatal patient in the neonatal intensive care unit (NICU), this strain was resistant to carbapenems including imipenem and meropenem. On January 23, 2014, another carbapenem-resistant $K$. pneumoniae strain (M2) was isolated from a stool sample obtained from another neonate in the same ward. We screened the rectal swab samples taken from patients in the NICU ward; simultaneously, environmental swabs of bed linen, stethoscopes, doorknobs, and water in the neonatal incubator, and the hand swabs obtained from doctors and nurses, were also collected. All swabs were inoculated on the Mueller-Hinton plates containing $2 \mu \mathrm{g} / \mathrm{mL}$ meropenem. The colonies that grew on the selection medium and clinical isolates with decreased susceptibility to carbapenems were picked and identified using a VITEK 2 Compact (bioMérieux, Marcy l'Etoile, France).

\section{Detection of phenotypes}

The production of carbapenemases was evaluated in all isolates using a Modified Hodge test [13] and Carba NP test [14], as previously described.

\section{Antimicrobial susceptibility testing}

MICs for antimicrobial agents were determined by using the microdilution susceptibility testing method, according to the guidelines of the CLSI [15]. The antibiotics tested included imipenem, meropenem, ceftazidime, aztreonam, piperacillin, piperacillin/tazobactam, tigecycline, levofloxacin, and amikacin. MIC results were interpreted as specified by CLSI [13], except for tigecycline, which was interpreted as defined by the US Food and Drug Administration (susceptible: MIC $\leq 2 \mathrm{mg} / \mathrm{L}$; resistant: MIC $\geq 8 \mathrm{mg} / \mathrm{L}$ ). Escherichia coli ATCC 25922 was used as quality control.

\section{Detection of drug-resistant genes}

Bacterial chromosomal DNA was obtained from clinical strains and transconjugants with a TIANamp Bacterial DNA Kit according to the manufacturer's instructions (TIANGEN BIOTECH, Beijing, China). PCR and DNA sequence analysis were performed to confirm the presence of drug-resistant genes. The primers used in this study were described previously $[3,16]$. $\beta$-lactamase genes, including, Ambler class A $\left(b l a_{\mathrm{CTX}-\mathrm{M}}, b l a_{\mathrm{TEM}}\right.$, $b l a_{\mathrm{SHV}}, b l a_{\mathrm{KPO}} b l a_{\mathrm{IMI}}$ and $\left.b l a_{\mathrm{GES}}\right)$, class $\mathrm{B}\left(b l a_{\mathrm{VIM}}\right.$, $b l a_{\mathrm{IMP}}, b l a_{\mathrm{NDM}}$, and $\left.b l a_{\mathrm{SPM}}\right)$, class C $\left(b l a_{\mathrm{CMY}}, b l a_{\mathrm{ACT}-1}\right.$, and $\left.b l a_{\text {DHA-1 }}\right)$, and class D (bla $\left.a_{\mathrm{OXA}-48}\right)$ were detected in all clinical isolates and their transconjugants. Moreover, genes related to quinolone activity including $q n r A$, $q n r B$, and qurS, integron genes and the aac gene were also detected. Products were sequenced on an ABI PRISM 3730AXL sequencer analyzer and compared with the reported sequences from GenBank.

\section{Molecular typing}

NDM-1-producing strains were genotyped by using MLST and PFGE. Seven housekeeping genes (gapA, infB, $m d h$, pgi, phoE, rpoB, and tonB) were amplified according to the protocol described on the MLST website [17]. PFGE were performed according to the procedure described by Pulse Net from the website of the Centers for Disease Control and Prevention [18]. Salmonella enterica serotype $\mathrm{H} 9812$ was used as a marker. The $\mathrm{XbaI}$ restriction patterns were analyzed and interpreted according to the criteria of Tenover et al. [19].

\section{Analysis of plasmid and conjugation experiment}

In order to determine whether carbapenem resistance was transferable, a conjugation experiment was carried 
out in mixed broth cultures. Escherchia coli $\mathrm{J} 53\left(\mathrm{Az}^{\mathrm{R}}\right)$ was used as the recipient strain. Test strains and the recipient strain were grown separately overnight in Luria-Bertani broth at $35^{\circ} \mathrm{C}$ with shaking. Cultures $(2 \mathrm{ml})$ of test strains and recipient strains were mixed in a tube and incubated at $35{ }^{\circ} \mathrm{C}$ for $4 \mathrm{~h}$ with shaking. Then, $50 \mu \mathrm{L}$ of the mixture was placed on Mueller-Hinton agar containing $2 \mu \mathrm{g} / \mathrm{mL}$ meropenem and $200 \mathrm{mg} / \mathrm{L}$ sodium azide and incubated at $35^{\circ} \mathrm{C}$ for $20 \mathrm{~h}$. The colonies that grew on this medium were regarded as the products of successful conjugation and were picked up and identified using a VITEK 2 Compact. Plasmid DNA from donors and transformants were extracted with a TIANprep Plasmid Maxi Plasmid Kit according to the manufacturer's instructions (TIANGEN BIOTECH, Beijing, China) and was electrophoresed on $0.8 \%$ agarose gels at $100 \mathrm{~V}$ for $4 \mathrm{~h}$. The plasmid replicons of the $b l a_{\mathrm{NDM}-1}$-encoding plasmids were typed by using the PCR-based replicon typing method described previously [20].

\section{Results}

\section{Bacterial isolates}

Eighteen carbapenem-resistant $K$. pneumoniae strains (M1-M18) were isolated from 17 patients in a variety specimens including sputum, stool, and blood; and one carbapenem-resistant K. pneumoniae (M19) was detected in incubator water. The CRE outbreak was declared on March 31, 2014. Resistace screening was performed for all patients in the NICU until no further transmission was detected. All the 17 patients had received meropenem treatment as initial monotherapy, one patient died on February 10, 2014, while all others were recovered.

\section{Phenotypes and drug-resistant genes}

All strains (M1-M19) harbored $b l a_{\mathrm{NDM}-1}$, a carbapenemase-encoding gene. M3, M5, M8, M9, M17, M18, and M19 co-harbor another carbapenemase gene $b$ IMP-4 $_{\mathrm{IM}}$. Thirteen of 19 isolates showed positive phenotypic screening results for the Modified Hodge test, the positivity rate was $72 \%$, while the positive rate for the Carba-NP test was $100 \%$. Other $\beta$-lactamase genes were identified in 19 strains, including those of the $b l a_{\mathrm{TEM}}, b l a_{\text {СТX-M}}$, and $b l a_{\mathrm{SHV}}$. The $q n r$ and $a a c$ genes were also detected; no AmpC-like enzymes and integron genes were found. The remaining resistance genes that were evaluated were not detected. Details on these findings are shown in Table 1.

\section{Antimicrobial susceptibility testing}

Drug-resistance profiles were consistent between the 18 NDM-1-producing K. pneumoniae clinical isolates (M1M18) and the one NDM-1-producing $K$. pneumoniae strain obtained from incubator water (M19). All the 19 strains were highly resistant to the tested carbapenems, including meropenem and imipenem. The MIC values for meropenem were in the range of $32-128 \mu \mathrm{g} / \mathrm{mL}$ and those of imipenem ranged from 4 to $>128 \mu \mathrm{g} / \mathrm{mL}$. Nineteen strains exhibited discrepant-level resistance to aztreonam, six isolates were sensitive, seven isolates were intermediate, and the rest six were resistant. The MIC values for the other tested $\beta$-lactam antibiotics were high $(>128 \mu \mathrm{g} / \mathrm{mL})$ in all tested strains. Tigecycline exhibited potent activity against all tested strains, none tigecycline reisitant strain was detected. All the isolates remained susceptible to ciprofloxacin and amikacin. These results are summarized in Table 1.

\section{PFGE and MLST typing}

PFGE patterns of the XbaI DNA digests of 19 K. pneumoniae isolates were obtained. Gel images were input into BioNumerics and phylogenetic tree was built for cluster analysis (Fig. 1). PFGE revealed four cluster among 19 K. pneumoniae. One cluster of 16 closely related isolates was found that exhibited $>90 \%$ similarities. MLST analysis showed that all the $19 \mathrm{~K}$. pneumoniae strains identified here were defined as a single sequence type (ST105) with the allelic profile 2-3-2-1-1-4-18.

\section{Plasmid analysis and bacterial conjugation}

Carbapenems resistance was successfully transferred from all K. pneumoniae isolates to E. coli $\mathrm{J} 53\left(\mathrm{Az}^{\mathrm{R}}\right)$ by conjugation. The MIC values of the 19 transconjugants were tested, and all E. coli transconjugants exhibited significantly reduced carbapenem susceptibility to the tested carbapenems, including imipenem and meropenem, as compared to E. coli $\mathrm{J} 53\left(\mathrm{Az}^{\mathrm{R}}\right)$. Meanwhile, the transconjugants were resistant to $\beta$-lactam antibiotics, although not to aztreonam, and were susceptible to quinolones and aminoglycosides (Table 2). Analysis of plasmids harbored by M1-M19 and transconjugants revealed the presence of two plasmids (ca. 50 and ca. $2.3 \mathrm{~kb}$ ), while the transconjugants only acquired the ca. $50-\mathrm{kb}$ plasmid. PCR analysis confirmed that the plasmid present in transconjugants harbored both $b l a_{\mathrm{NDM}-1}$ and $b l a_{\mathrm{SHV}-1}$. PCR-based inc/rep typing method showed that FIA, FIB, $\mathrm{FIC}$, and $\mathrm{F}$ replicons were positive in all $b l a_{\mathrm{NDM}-1^{-}}$encoding plasmids, which belonged to IncFI incompatibility group.

\section{Discussion}

Emergence of NDM-1-producing Enterobacteriaceae have disseminated worldwide from the Indian subcontinent brought about problems regarding therapy and control. In China, plasmids encoding $b l a_{\mathrm{NDM}-1}$ have been identified in Enterobacteriaceae isolates in several regions including Beijing, Shanghai, Hong Kong, and 


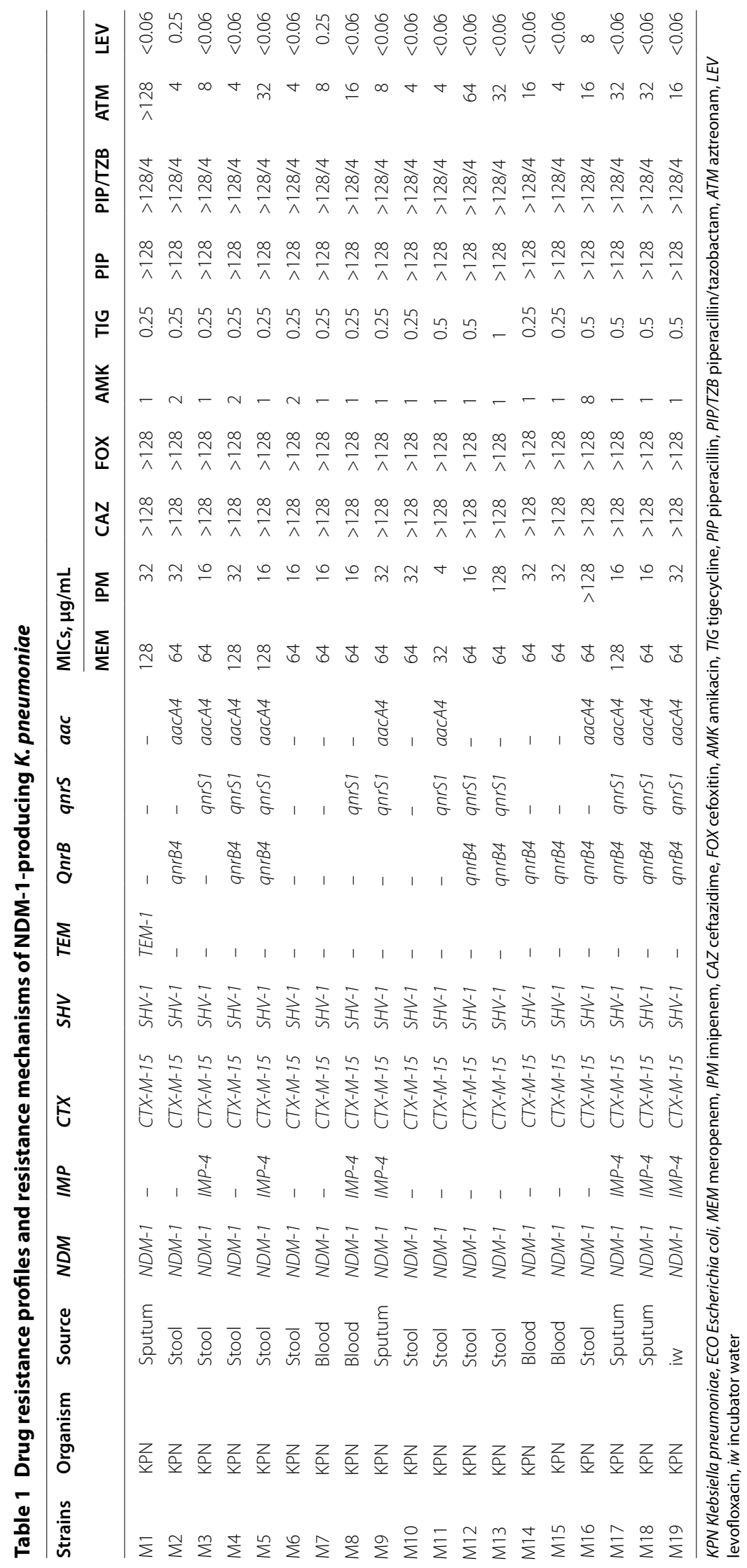




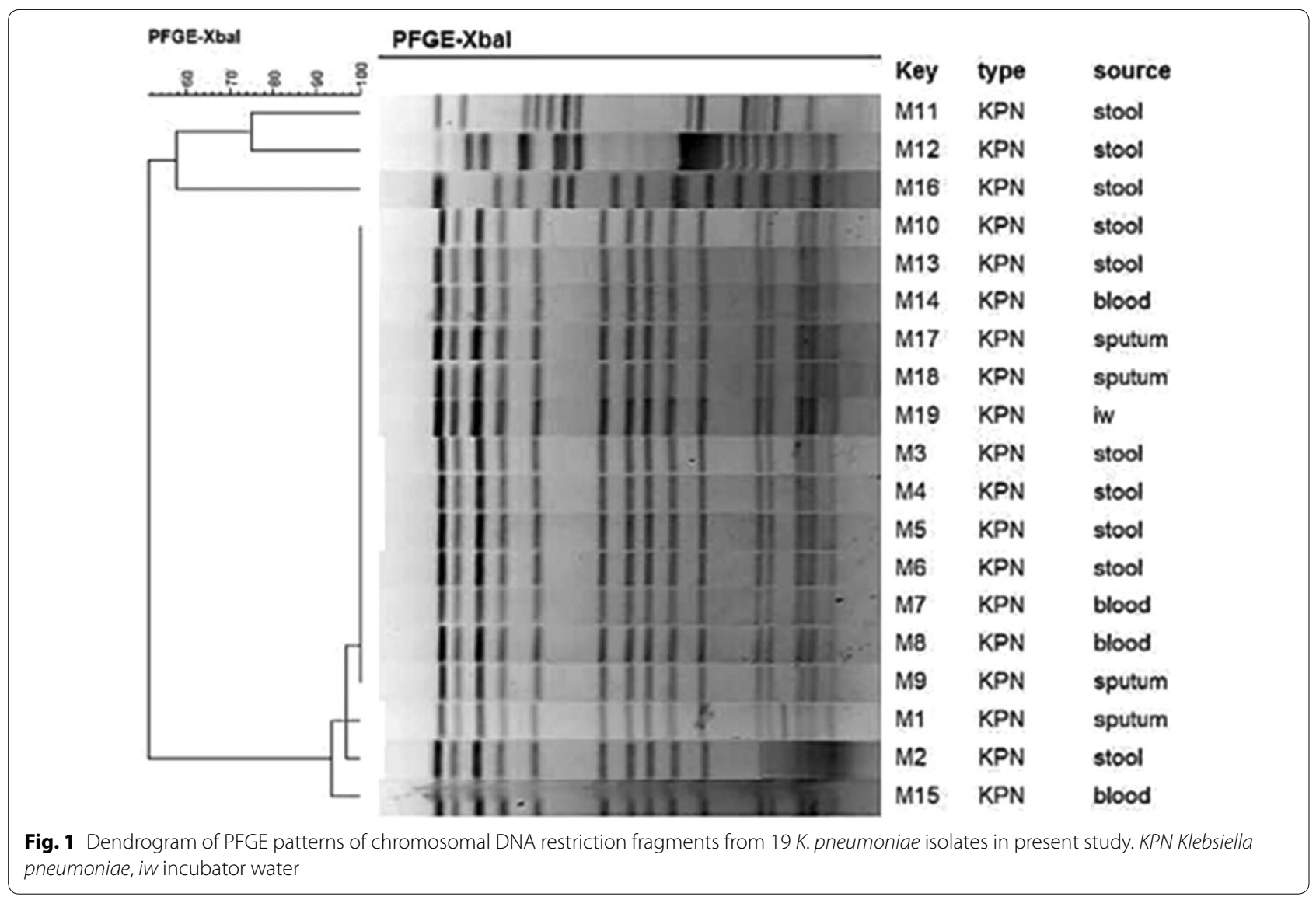

Shandong province, the size and Inc-type of the plasmids harbouring $b l a_{\mathrm{NDM}-1}$ were vary from $\sim 50$ to $\sim 336 \mathrm{~kb}$ including InX3, IncL/M, IncA/C, and IncN incompatibility group [11, 21-23]. In present study, the plasmid harbouring the $b l a_{\mathrm{NDM}-1}$ belonged to the IncFI-type, which were different from previously replicon type reported in China before. Plasmid replicon types were related to the dissemination of resistance genes [22]. Due to its presence in all the 19 CREs in this study, this IncFI plasmid may be responsible for the dissemination of the $b l a_{\mathrm{NDM}-1}$ in this area.

Moreover, dissemination of $b l a_{\mathrm{NDM}-1}$ is associated with MLST type [24]. NDM-1-producing $K$. pneumoniae have been reported in different countries, and belonged to various kinds of MLST types, including ST11, ST14, ST17, ST25, ST147, ST149, ST231, ST340, and ST1043 [24-29]. Our datas indicated that all 19 NDM-1-producing $K$. pneumoniae strains belong to the same type, viz., ST105, which was different from previous types reported before. PFGE analysis showed 4 clusters for 19 ST105 strains. Among them, one cluster of 16 closely related isolates was found that exhibited $\geq 90 \%$ similarities including the strains detected in incubator water. Thoese results suggested that 19 NDM-1-producing K. pneumoniaee strains were clonally related and easily spread to different patients in NICU ward, environmental reservoirs such as incubator water may contribute to the spread of these organisms within hospital. A previous research showed that $b l a_{\mathrm{NDM}-1}$ gene had disseminated in the NICU via different Gram Negative Bacilli (E. coli, A. baumannii, S. maltophilia and/or K. pneumoniae) harbouring bla $a_{\mathrm{NDM}-1}$ [30]. However, it is unclear how the $b l a_{\mathrm{NDM}-1}$ was introduced into the NICU ward in Kunming City Maternal and Child health Hospital. We suspect that isolates in this study represent a novel ST and that autochthonous clones are locally acquiring plasmids carrying the $b l a_{\mathrm{NDM}-1}$, as has been reported previously [27], more research would be needed to uncover it.

In addition, the average days for hospitalization in Kunming City Maternal and Child health Hospital is 6.06 days at present. However, the average hospital stay of the 17 patients including in this study was 18.9 days (more than trebled of average days of hospitalization in this hospital), prolonged hospitalization may contribute to spreading of the ST105 strains in NICU ward.

The $b l a_{\mathrm{IMP}-4}$ carbapenemase-encoding genes have also been detected in the part of NDM-1-producing strains (7/19). Klebsiella pneumoniae strains co-harbouring 
Table 2 The results of antibiotic susceptibility testing of transconjugants in present study

\begin{tabular}{|c|c|c|c|c|c|c|c|c|c|c|}
\hline Strains & MEM & IPM & CAZ & FOX & AMK & TIG & PIP & PIP/TZP & ATM & LEV \\
\hline M1-J53 & 32 & 4 & 64 & 64 & 1 & $<0.125$ & $>128$ & $>128 / 4$ & 0.5 & $<0.06$ \\
\hline M2-J53 & 32 & 4 & 64 & 64 & 1 & $<0.125$ & $>128$ & $>128 / 4$ & 0.5 & $<0.06$ \\
\hline M3-J53 & 16 & 4 & 128 & 64 & 1 & $<0.125$ & $>128$ & $>128 / 4$ & 0.5 & $<0.06$ \\
\hline M4-J53 & 32 & 16 & 128 & 64 & 1 & $<0.125$ & $>128$ & $>128 / 4$ & 0.5 & $<0.06$ \\
\hline M5-J53 & 32 & 4 & 64 & 64 & 1 & $<0.125$ & $>128$ & $>128 / 4$ & 0.5 & $<0.06$ \\
\hline M6-J53 & 32 & 4 & 64 & 64 & 1 & $<0.125$ & $>128$ & $>128 / 4$ & 0.5 & $<0.06$ \\
\hline M7-J53 & 32 & 8 & 64 & 64 & 1 & $<0.125$ & $>128$ & $>128 / 4$ & 0.5 & $<0.06$ \\
\hline M8-J53 & 32 & 4 & 64 & 64 & 1 & $<0.125$ & $>128$ & $>128 / 4$ & 0.5 & $<0.06$ \\
\hline M9-J53 & 32 & 4 & 128 & 64 & 1 & $<0.125$ & $>128$ & $>128 / 4$ & 0.5 & $<0.06$ \\
\hline M10-J53 & 32 & 4 & 64 & 64 & 1 & $<0.125$ & $>128$ & $>128 / 4$ & 0.5 & $<0.06$ \\
\hline M11-J53 & 16 & 4 & $>128$ & 64 & 1 & $<0.125$ & $>128$ & $>128 / 4$ & 0.5 & $<0.06$ \\
\hline M12-J53 & 32 & 8 & 64 & 64 & 1 & $<0.125$ & $>128$ & $>128 / 4$ & 0.5 & $<0.06$ \\
\hline M13-J53 & 32 & 4 & 64 & 64 & 1 & $<0.125$ & $>128$ & $>128 / 4$ & 4 & $<0.06$ \\
\hline M14-J53 & 32 & 4 & 64 & 64 & 1 & $<0.125$ & $>128$ & $>128 / 4$ & 2 & $<0.06$ \\
\hline M15-J53 & 32 & 8 & 64 & 64 & 1 & $<0.125$ & $>128$ & $>128 / 4$ & 0.5 & $<0.06$ \\
\hline M16-J53 & 32 & 4 & 64 & 64 & 1 & $<0.125$ & $>128$ & $>128 / 4$ & 0.5 & $<0.06$ \\
\hline M17-J53 & 32 & 4 & 32 & 64 & 1 & $<0.125$ & $>128$ & $>128 / 4$ & 0.5 & $<0.06$ \\
\hline M18-J53 & 32 & 16 & 64 & 64 & 1 & $<0.125$ & $>128$ & $>128 / 4$ & 4 & $<0.06$ \\
\hline M19-J53 & 32 & 4 & 64 & 64 & 1 & $<0.125$ & $>128$ & $>128 / 4$ & 4 & $<0.06$ \\
\hline E. coli J53 & $<0.125$ & $<0.125$ & $<0.125$ & 8 & 1 & $<0.125$ & 4 & $2 / 4$ & 0.5 & $<0.06$ \\
\hline
\end{tabular}

MEM meropenem, IPM imipenem, CAZ ceftazidime, FOX cefoxitin, AMK amikacin, TIG tigecycline, PIP piperacillin, PIP/TZB piperacillin/tazobactam, ATM aztreonam, LEV levofloxacin

$b l a_{\mathrm{NDM}-1}$ and $b l a_{\mathrm{IMP}-4}$ have been identified in China previously, when they were found to be colocalized on a ca. 300-kb plasmid [31]. Plasmid analysis in this study showed that transconjugants acquired a ca. $50-\mathrm{kb}$ plasmid. We analyzed the genomic DNA and plasmid DNA of 19 transconjugants by PCR, and confirmed only the presence of the $b l a_{\mathrm{NDM}-1}$ and $b l a_{\mathrm{SHV}-1}$. This suggested that $b l a_{\mathrm{IMP}-4}$ was not on the ca. 50-kb IncFI plasmid along with $b l a_{\mathrm{NDM}-1}$. We speculate that the $b l a_{\mathrm{IMP}-4}$ gene may lie on the chromosome or a high molecular weight non-conjugative plasmid of which was not detected by the methodology used, further research was needed to uncover it. $B l a_{\mathrm{CTX}-\mathrm{M}-15}$ and $b l a_{\mathrm{NDM}-1}$ have a common origin in the Indian subcontinent [25] and $b l a_{\mathrm{CTX}-\mathrm{M}-15}$ had been identified in the NDM-1-producing strains, irrespective of whether the genes were located on the same plasmid [32,33] or not [34]. Our study showed the presence of $b l a_{\mathrm{CTX}-\mathrm{M}-15}$ along with $b l a_{\mathrm{NDM}-1}$ in all strains, but none of the 17 patients or their family had any epidemiological link to the Indian subcontinent. AmpCs always been detected with NMD-1 producers [28], but $b l a_{\text {ACT-1, }}$, $b l a_{\mathrm{CMY}}$, and $b l a_{\mathrm{DHA}-1}$ were not been found in this study. Although qnrB4, qnrS1 and aacA4 were detected in some strains, levofloxacin and amikacin maintained a good antibacterial activity in vitro.
Nineteen transconjugants showed increased MIC values for the tested carbapenems as compared with $E$. coli J53 $\left(\mathrm{Az}^{\mathrm{R}}\right)$. The MIC values of 19 transconjugants for meropenem and imipenem ranged from 16 to $32 \mu \mathrm{g} / \mathrm{mL}$ and 4 to $16 \mu \mathrm{g} / \mathrm{mL}$ respectively, which was more than fourfold higher than those of $E$. coli J53. Due to the transconjugants only acquired $b l a_{\mathrm{NDM}-1}$ and $b l a_{\mathrm{SHV}-1}$, we concluded that $b l a_{\mathrm{NDM}-1}$ was primarily responsible for the high MIC values of carbapenems. In comprehensive consideration of both the MIC values of clinical isolates (M1-M18) and their transconjugants, $b l a_{\mathrm{IMP}-4}$ and $b l a_{\mathrm{CTX}-\mathrm{M}-15}$ also played a role in conferring resistance to the carbapenems.

\section{Conclusions}

Hospital environment such as incubator water may be the diffusion reservoirs of NDM-1-producing bacteria. Personal contact between the caregivers and the patients hospitalized in the same ward is the most likely transmission route. However, it can not determine how this clone was introduced into the hospital, ST105 K. pneumoniae may have been spreading in hospitals in the region and their prevalence may be increasing. Therefore, nosocomial surveillance system should play a more important role in the infection control to limit the spread of NDM1-producing pathogens. 


\section{Abbreviations}

CLSI: Clinical and Laboratory Standards Institute; CPE: carbapenemase-producing Enterobacteriaceae; CRE: carbapenem-resistant Enterobacteriaceae; ESBL: extended-spectrum $\beta$-lactamase; MDR: multidrug-resistant; MLST: multilocussequence typing; NICU: neonatal intensive care unit; PFGE: pulsed field gel electrophoresis.

\section{Authors' contributions}

XX designed the study; RZ drafted the first version of this manuscript; $Q Z$ collected the isolates and clinical informations; YG performed the PFGE; RZ and QZ preformed the antimicrobial susceptibility test and conjugation experiment; RZ, YF, LL, AZ, YZ, and XY carried out the molecular biology experiments. All authors read and approved the final manuscript.

\section{Author details}

${ }^{1}$ Faculty of Environmental Science and Engineering, Kunming University of Science and Technology, Kunming, Yunnan, China. ${ }^{2}$ Faculty of Life Science and Technology, Kunming University of Science and Technology, No. 727 Jing Ming Road, Chenggong District, Kunming, Yunnan, China. ${ }^{3}$ Department of Clinical Laboratory, First People's Hospital of Yunnan province, Kunming, Yunnan, China. ${ }^{4}$ Department of Clinical Laboratory, Kunming City Maternal and Child health Hospital, Kunming, Yunnan, China. ${ }^{5}$ Yunnan Center for Disease Control and Prevention, Kunming, Yunnan, China.

\section{Acknowledgements}

Escherchia coli 553 was a kind gift from Prof. Yang Qing, the First Affiliated Hospital of Zhejiang University. This work was supported by Yunnan Science and Technology Commission (2013FB205, 2015BC001) from Yunnan provincial Science and Technology Department and Kunming Medical University.

\section{Competing interests}

The authors declare that they have no competing interests.

Received: 14 August 2015 Accepted: 3 February 2016

Published online: 19 February 2016

\section{References}

1. Gaynes R, Edwards JR, National Nosocomial Infections Surveillance S. Overview of nosocomial infections caused by gram-negative bacilli. Clin Infect Dis. 2005;41(6):848-54. doi:10.1086/432803.

2. Walsh TR, Toleman MA. The emergence of pan-resistant Gram-negative pathogens merits a rapid global political response. J Antimicrob Chemother. 2012;67(1):1-3. doi:10.1093/jac/dkr378.

3. Li H, Zhang J, Liu Y, Zheng R, Chen H, Wang X, et al. Molecular characteristics of carbapenemase-producing Enterobacteriaceae in China from 2008 to 2011: predominance of KPC-2 enzyme. Diagn Microbiol Infect Dis. 2014;78(1):63-5. doi:10.1016/j.diagmicrobio.2013.10.002.

4. Dortet L, Poirel L, Nordmann P. Worldwide dissemination of the NDMtype carbapenemases in gram-negative bacteria. BioMed Res Int. 2014;2014:249856. doi:10.1155/2014/249856.

5. Yong D, Toleman MA, Giske CG, Cho HS, Sundman K, Lee K, et al. Characterization of a new metallo- $\beta$-lactamase gene, blaNDM- 1 , and a novel erythromycin esterase gene carried on a unique genetic structure in Klebsiella pneumoniae sequence type 14 from India. Antimicrob Agents Chemother. 2009:53(12):5046-54.

6. Tzouvelekis LS, Markogiannakis A, Psichogiou M, Tassios PT, Daikos GL. Carbapenemases in Klebsiella pneumoniae and other Enterobacteriaceae: an evolving crisis of global dimensions. Clin Microbiol Rev. 2012:25(4):682-707. doi:10.1128/CMR.05035-11.

7. Barman S, Chatterjee S, Chowdhury G, Ramamurthy T, Niyogi SK, Kumar $R$, et al. Plasmid-mediated streptomycin and sulfamethoxazole resistance in Shigella flexneri 3a. Int J Antimicrob Agents. 2010;36(4):348-51. doi:10.1016/j.jiantimicag.2010.06.037.

8. Coque TM, Novais A, Carattoli A, Poirel L, Pitout J, Peixe L, et al. Dissemination of clonally related Escherichia coli strains expressing extended-spectrum beta-lactamase CTX-M-15. Emerg Infect Dis. 2008;14(2):195-200. doi:10.3201/eid1402.070350.
9. Kumarasamy KK, Toleman MA, Walsh TR, Bagaria J, Butt F, Balakrishnan $R$, et al. Emergence of a new antibiotic resistance mechanism in India, Pakistan, and the UK: a molecular, biological, and epidemiological study. Lancet Infect Dis. 2010;10(9):597-602.

10. Jarvis WR, Munn VP, Highsmith AK, Culver DH, Hughes JM. The epidemiology of nosocomial infections caused by Klebsiella pneumoniae. Infect Control. 1985:6(2):68-74.

11. Jin Y, Shao C, Li J, Fan H, Bai Y, Wang Y. Outbreak of multidrug resistant NDM-1-producing Klebsiella pneumoniae from a neonatal unit in Shandong Province, China. PLoS One. 2015;10(3):e0119571. doi:10.1371/ journal.pone.0119571.

12. Wang X, Xu X, Li Z, Chen H, Wang Q, Yang P, et al. An outbreak of a nosocomial NDM-1-producing Klebsiella pneumoniae ST147 at a teaching hospital in mainland China. Microbial Drug Res. 2014;20(2):144-9. doi:10.1089/mdr.2013.0100.

13. CLSI. Performance standards for antimicrobial susceptibility testing; 23rd informational supplement. M100-S23: Clinical and Laboratory Standards Institute, Wayne, PA. 2013.

14. Nordmann P, Poirel L, Dortet L. Rapid detection of carbapenemaseproducing Enterobacteriaceae. Emerg Infect Dis. 2012;18(9):1503.

15. CLSI. Methods for dilution antimicrobial susceptibility tests for bacteria that grow aerobically, 9th ed. Approved standard M07-A9: Clinical and Laboratory Standards Institute, Wayne, PA. 2012.

16. Yang $Q$, Wang $H$, Sun $H$, Chen $H$, Xu Y, Chen M. Phenotypic and genotypic characterization of Enterobacteriaceae with decreased susceptibility to carbapenems: results from large hospital-based surveillance studies in China. Antimicrob Agents Chemother. 2010;54(1):573-7. doi:10.1128/AAC.01099-09.

17. Institut pasteur MLST databases. http://bigsdb.web.pasteur.fr/klebsiella/ klebsiella.html. Accessed 19 Sep 2014.

18. Centers for Disease Control and Prevention. http://www.cdc.gov/ pulsenet/protocols.htm. Accessed 20 May 2008.

19. Tenover FC, Arbeit RD, Goering RV, Mickelsen PA, Murray BE, Persing DH, et al. Interpreting chromosomal DNA restriction patterns produced by pulsed-field gel electrophoresis: criteria for bacterial strain typing. J Clin Microbiol. 1995:33(9):2233-9.

20. Carattoli A, Bertini A, Villa L, Falbo V, Hopkins KL, Threlfall EJ. Identification of plasmids by PCR-based replicon typing. J Microbiol Methods. 2005;63(3):219-28. doi:10.1016/j.mimet.2005.03.018.

21. Ho PL, Lo WU, Yeung MK, Lin CH, Chow KH, Ang I, et al. Complete sequencing of pNDM-HK encoding NDM-1 carbapenemase from a multidrug-resistant Escherichia coli strain isolated in Hong Kong. PLoS ONE. 2011;6(3):e17989.

22. Qu H, Wang X, Ni Y, Liu J, Tan R, Huang J, et al. NDM-1-producing Enterobacteriaceae in a teaching hospital in Shanghai, China: IncX3-type plasmids may contribute to the dissemination of bla. Int J Infect Dis. 2015;34:8-13. doi:10.1016/j.jijid.2015.02.020.

23. Zhou G, Guo S, Luo Y, Ye L, Song Y, Sun G, et al. NDM-1-producing Strains, Family Enterobacteriaceae, in Hospital, Beijing, China. Emerg Infect Dis. 2014:20(2):340.

24. Giske CG, Froding I, Hasan CM, Turlej-Rogacka A, Toleman M, Livermore $D$, et al. Diverse sequence types of Klebsiella pneumoniae contribute to the dissemination of blaNDM-1 in India, Sweden, and the United Kingdom. Antimicrob Agents Chemother. 2012;56(5):2735-8. doi:10.1128/ AAC.06142-11.

25. Oteo J, Domingo-Garcia D, Fernandez-Romero S, Saez D, Guiu A Cuevas $\mathrm{O}$, et al. Abdominal abscess due to NDM-1-producing Klebsiella pneumoniae in Spain. J Med Microbiol. 2012;61(Pt 6):864-7. doi:10.1099/ jmm.0.043190-0.

26. Voulgari E, Gartzonika C, Vrioni G, Politi L, Priavali E, Levidiotou-Stefanou S, et al. The Balkan region: NDM-1-producing Klebsiella pneumoniae ST11 clonal strain causing outbreaks in Greece. The Journal of antimicrobial chemotherapy. 2014;69(8):2091-7. doi:10.1093/jac/dku105.

27. Escobar Perez JA, Olarte Escobar NM, Castro-Cardozo B, Valderrama Marquez IA, Garzon Aguilar MI, Martinez de la Barrera L, et al. Outbreak of NDM-1-producing Klebsiella pneumoniae in a neonatal unit in Colombia. Antimicrob Agents Chemother. 2013;57(4):1957-60. doi:10.1128/ AAC.01447-12.

28. Poirel L, Dortet L, Bernabeu S, Nordmann P. Genetic features of blaNDM-1-positive Enterobacteriaceae. Antimicrob Agents Chemother. 2011;55(11):5403-7. doi:10.1128/AAC.00585-11. 
29. Pasteran F, Albornoz E, Faccone D, Gomez S, Valenzuela C, Morales M, et al. Emergence of NDM-1-producing Klebsiella pneumoniae in Guatemala. J Antimicrob Chemother. 2012;67(7):1795-7. doi:10.1093/jac/ dks101.

30. Roy S, Singh AK, Viswanathan R, Nandy RK, Basu S. Transmission of imipenem resistance determinants during the course of an outbreak of NDM-1 Escherichia coli in a sick newborn care unit. J Antimicrob Chemother. 2011;66(12):2773-80. doi:10.1093/jac/dkr376.

31. Chen Z, Wang Y, Tian L, Zhu X, Li L, Zhang B, et al. First Report in China of Enterobacteriaceae clinical isolates coharboring blaNDM-1 and blalMP-4 drug resistance genes. Microbial Drug Res. 2014. doi:10.1089/ mdr.2014.0087.
32. Yoo JS, Kim HM, Koo HS, Yang JW, Yoo Jl, Kim HS, et al. Nosocomial transmission of NDM-1-producing Escherichia coli ST101 in a Korean hospital. J Antimicrob Chemother. 2013;68(9):2170-2. doi:10.1093/jac/dkt126.

33. Solé M, Pitart C, Roca I, Fàbrega A, Salvador P, Muñoz L, et al. First description of an Escherichia coli strain producing NDM-1 carbapenemase in Spain. Antimicrob Agents Chemother. 2011;55(9):4402.

34. Poirel L, Revathi G, Bernabeu S, Nordmann P. Detection of NDM-1-producing Klebsiella pneumoniae in Kenya. Antimicrob Agents Chemother. 2011;55(2):934-6.
Submit your next manuscript to BioMed Central and we will help you at every step:

- We accept pre-submission inquiries

- Our selector tool helps you to find the most relevant journal

- We provide round the clock customer support

- Convenient online submission

- Thorough peer review

- Inclusion in PubMed and all major indexing services

- Maximum visibility for your research

Submit your manuscript at www.biomedcentral.com/submit
(O) BioMed Central 